\title{
Clinical study of modulated electro-hyperthermia for advanced metastatic breast cancer
}

\author{
TAKUYA NAGATA ${ }^{1}$, MASAHIKO KANAMORI ${ }^{2}$, SHINICHI SEKINE $^{3}$, \\ MIE ARAI $^{4}$, MAKOTO MORIYAMA ${ }^{5}$ and TSUTOMU FUJII ${ }^{6}$ \\ ${ }^{1}$ Department of Surgery, Toho University Ohashi Medical Center, Tokyo 153-8515; ${ }^{2}$ Department of Human Science, \\ University of Toyama, Toyama 930-0194; ${ }^{3}$ Department of Surgery, Kamiichi General Hospital, Toyama 930-0391; \\ ${ }^{4}$ Department of Surgery, Toyama Nishi General Hospital, Toyama 939-2716; ${ }^{5}$ Department of Surgery, Tomei Atsugi Hospital, \\ Kanagawa 243-8571; ${ }^{6}$ Department of Science and Surgery, University of Toyama, Toyama 930-0194, Japan
}

Received April 28, 2020; Accepted March 5, 2021

DOI: $10.3892 / \mathrm{mco} .2021 .2265$

\begin{abstract}
Modulated electro-hyperthermia (mEHT) is a new treatment modality developed to overcome the problems associated with traditional hyperthermia; mEHT uses a precise impedance-matched system and modulated radiofrequency current flow to malignant tumors. It selects the malignant cells based on their biophysical differences, due to their high metabolic rate, individual (autonomic) behavior and membrane status. The aim of the present study was to report the outcomes of mEHT in the treatment of advanced breast cancer. mEHT was examined in 10 patients with advanced metastatic breast cancer and recurrent disease, who were considered incurable by standard therapy protocols. Of the 10 patients, partial response was achieved in 3 , disease stability in 3, and progressive disease in 4; however, their quality of life was improved based on their subjective reports. No adverse effects were observed in any of the 10 patients. The present study demonstrated the feasibility of mEHT as a possible therapy for advanced breast cancer cases when standard therapies fail. Moreover, mEHT had no side effects and may be combined with various treatments for long-term therapy.
\end{abstract}

\section{Introduction}

For patients with locally advanced and/or recurrent breast cancer, many multidisciplinary approaches, including hormonal therapy (1), irradiation (2), surgery (3), chemotherapy (4) and molecular target treatment (5), are used. However, patients with advanced breast cancer receiving prolonged periodic standard treatments may become refractory to many of them, thereby limiting the application of these therapeutic modalities. Moreover, the adverse effects of

Correspondence to: Professor Masahiko Kanamori, Department of Human Science, University of Toyama, 2630 Sugitani, Toyama 930-0194, Japan

E-mail: kanamori@med.u-toyama.ac.jp

Key words: breast cancer, hyperthermia, irradiation, chemotherapy therapy, such as general fatigue, multiple organ failure (lung, liver, kidney, bone, etc.) and/or pancytopenia (particularly neutrophil and platelet deficiency) may affect the general condition of the patient. As a result, it may be difficult or impossible to continue curative therapy. In case of widespread metastases, the risk is even higher.

Hyperthermia is one of the conventional forms of cancer therapy that forces the tumor to become necrotic due to absorbed heat. The dose is expressed in cumulative equivalent min for necrotic processes at $43^{\circ} \mathrm{C}\left(\mathrm{CEM} 43^{\circ} \mathrm{CTx}\right)(6)$ and is calibrated for necrotic cell death at $43^{\circ} \mathrm{C}$ in $\mathrm{x}$ percentage of the homogeneity of the actually measured $\mathrm{T}$ temperature. The missing technology of the isothermal heating of the heterogenic tumor mass and the necessity of performing in-depth temperature measurements of the tumor makes the treatment complicated, and insufficient results prevent its widespread acceptance in the clinic (7). Therefore, our hospital conducts clinical studies using modulated electro-hyperthermia (mEHT) as one of the new options for the multidisciplinary treatment of advanced cancer. $\operatorname{mEHT}(8,9)$, is a new treatment modality developed to overcome the problems of traditional hyperthermia; it uses a precise impedance-matched system and modulated radiofrequency current flow to malignant tumors (10), which select the malignant cells based on their biophysical differences, due to their high metabolic rate, individual (autonomic) behavior and membrane status (11).

mEHT has been successfully used for the treatment of patients with various stages and forms of breast cancer, including in a retrospective study with 103 patients (12), a case of primary leiomyosarcoma of the breast following salvage hyperthermia and pazopanib therapy (13), and a case of long-term survival of a breast cancer patient with extensive liver metastases upon immunotherapy and virotherapy (14).

The aim of this study was to present a clinical study of patients with advanced metastatic breast cancer who fail to respond to standard conventional therapies.

\section{Patients and methods}

Patients. Ten patients with advanced or recurrent breast cancer participated in the present study since November 2015. All patients had undergone conventional therapies following 
standard protocols for breast cancer. Patients received hormonal therapy, external irradiation, surgery, various chemotherapies, targeted molecular treatment, and other available state of the art therapies (15). The selected patients were treated with mEHT coupled with adjuvant therapies (chemotherapy, hormone therapy or irradiation) when possible (6 cases); in case of complete failure of conventional methods, monotherapy was used (4 cases). The adjuvant therapies were trastuzumab emtansine (TDM-1; 1 case), mammalian target of rapamycin (mTOR; 3 cases), eribulin (1 case), irradiation (1 case) and fulvestrant ( 1 case). The study was approved by the local ethics committee of the University of Toyama (approval no. 26-13), and the patients provided written consent for the treatment, as well as for the research and publication of their data and images.

Procedure of $m E H T$. mEHT was performed twice a week in 7 patients and thrice a week in the other 3 . The session lasted for $\sim 60 \mathrm{~min}$, with at least 1 day in between. The treatment was performed using the EHY2000+ device (Oncotherm Kft.). The electrode used was $30 \mathrm{~cm}$ in diameter. Patients were placed in the supine position on the water mattress of the treatment bed. A step-up heating protocol was used, starting with $60 \mathrm{~W}$, which was then increased to $140 \mathrm{~W}$. The average number of treatments performed per patient was 48.6 (range, 8-90). The average dose of 374.6 (range, 371-376) kJ was administered.

Procedure and display of the analytical results. The endpoint of the study was local control (response rate). A follow-up examination of local control was conducted via inspection, computed tomography (CT), or magnetic resonance imaging, and was compared with that at baseline before the start of the mEHT treatment process. The age, estrogen receptor $(\mathrm{ER}) /$ progesterone receptor $(\mathrm{PgR}) /$ human epidermal growth factor receptor type 2 (HER2) status, actual status of metastases, and pretreatment for each patient are shown in Table I. The number and duration of mEHT sessions and the total amount of mEHT energy delivered to each patient are summarized in Table II. The complementary therapies and local responses are shown in Table III. The statistical analysis results are shown in Table IV.

Statistical analysis. The comparison of the distribution between the two groups of partial response (PR)+stable disease (SD) cases and progressive disease (PD) cases was conducted using unpaired t-test for continuous variables [age, total mEHT, $\mathrm{mEHT} / \mathrm{w}, \mathrm{mEHT}$ period, $\mathrm{mEHT}$ dose, pre-treatment, pre-CT, pre-carcinoembryonic antigen (CEA) and post-CEA] and the Mann-Whitney test for categorical variables (Stage). $\mathrm{P}<0.05$ was considered to indicate a statistically significant difference. All analyses were performed using the JMP15.0 software.

\section{Results}

Statistics of mEHT. Out of the 10 cases registered, 5 were stage 3 or 4 preoperatively (Table I). The ER status was positive in all cases, and HER 2 was positive in 1 case. In 9/10 cases, some treatments were performed before mEHT; however, due to the lack of a satisfactory antitumor effect, mEHT was performed or combined with other treatments (Table I). Case 2 received the most treatments prior to $\mathrm{mEHT}$, including two types of postoperative adjuvant chemotherapy, 5 types of chemotherapy for tumor recurrence, two types of hormone therapy, and irradiation. In addition, Case 2 received two types of chemotherapy in combination with mEHT (Table III). On the other hand, Case 10 received no treatment prior to $\mathrm{mEHT}$, following the patient's request. The statistics of the mEHT are shown in Table II. As a result, 8-90 mEHTs were performed. The decision to discontinue was entirely based on the request of the patient; the most common reason identified was difficulty in continuing the treatment. Case 7 underwent mEHT only 8 times. The reason for this was that the combined use of irradiation and mEHT reduced the metastatic skin cancer to PR; the patient hoped that mEHT would be terminated at the same time as the termination of irradiation. There were no apparent complications during mEHT.

Clinical estimation of the PD case. A summary of the local responses is presented in Table III. Patients felt comfortable with warming around the targeted area during treatment. The elevated body temperature observed was mild, and some patients presented with sweating without discomfort. In addition, there were no adverse effects, such as skin blisters, erythema, or dermatitis. PR was achieved in 3/10 (30\%) patients, and so was SD. A total of $4 / 10$ patients (40\%) showed PD. All 3 patients (cases 2,4 and 6) that were treated with a combination of $\mathrm{mEHT}$ and mTOR achieved PD. They had multiple-organ metastases from the breast cancer and had undergone multiple sessions of mEHT (46-90). Only case 2 received anthracycline and taxane for the treatment of breast cancer. Cases 4 and 6 refused chemotherapy and only approved the use of mTOR, which has relatively few side effects, such as hair loss and malaise. Therefore, these cases might have deviated from the usual treatment for advanced breast cancer and do not indicate a low therapeutic effect of the combination of mEHT and mTOR. However, 2/3 PR patients exhibited a re-increase in tumor size after the follow-up period. By contrast, another patient recovered and underwent curative surgery. At the time of writing, she was still alive with no signs of recurrence ( 9 months after initial mEHT therapy). A total of 4 patients judged as PD exhibited worsening of the local tumor and metastases. Three patients died of cancer during (2 patients) or after the completion of $\mathrm{mEHT}$ (1 patient). Case 2 was a 66-year-old woman. Bt+Ax was performed in the right breast. After administering two types of postoperative adjuvant chemotherapy, hormone therapy was performed. Five years after the operation, lung, liver and bone metastases occurred. Following recurrence, seven types of treatment were performed (five types of chemotherapy, one type of hormone therapy, and radiation therapy). In addition, case 2 received two types of chemotherapy in combination with 90 sessions of mEHT for 30 weeks. The tumor did not grow until 24 weeks after the start of treatment, but thereafter, lung metastasis gradually worsened, with the eventual occurrence of pleural effusion. Due to dyspnea, the patient could not visit the hospital; therefore, mEHT was discontinued. Three months later, the patient died of cancerous pleurisy. Case 5 was a 74-year-old woman. Bt+SLN was performed for left breast cancer. She continued hormone therapy following surgery. Three years after the operation, liver, bone and lymph node metastases occurred. Two types of chemotherapy, activated autologous lymphocyte therapy and 
Table I. Patient statistics and metastasis.

\begin{tabular}{rccccccccrrr}
\hline Case & Age, years & Surgery & Stage & ER & PgR & HER2 & Metastasis & CT & HT & RT & Total \\
\hline 1 & 58 & Bp+Ax & $2 \mathrm{~A}$ & + & + & + & Skin, lung, LN & 4 & 1 & 1 & 6 \\
2 & 66 & Bt+Ax & $3 \mathrm{~A}$ & + & + & - & Lung, liver, bone & 7 & 2 & 1 & 10 \\
3 & 63 & Bt+Ax & $2 \mathrm{~B}$ & + & + & - & Lung, LN & 0 & 2 & 0 & 2 \\
4 & 45 & Bp+Ax & $2 \mathrm{~B}$ & + & + & - & Bone, LN & 1 & 2 & 2 & 5 \\
5 & 74 & Bt+SLN & 1 & + & + & - & Liver, bone, LN & 2 & 1 & 0 & 3 \\
6 & 68 & $(-)$ & 4 & + & - & - & Lung, liver, bone & 0 & 4 & 2 \\
7 & 75 & $(-)$ & 4 & + & - & - & Skin, lung & 3 & 0 & 1 \\
8 & 49 & Bt+Ax & 4 & + & + & - & Lung & 4 \\
9 & 66 & Bt+Ax & $2 \mathrm{~A}$ & + & - & - & Lung, liver, bone & 4 & 2 & 1 \\
10 & 71 & Bt+Ax & $3 \mathrm{~A}$ & + & + & - & Skin, muscle & 0 & 0 & 0 \\
\hline
\end{tabular}

Bp, partial mastectomy; Bt, total mastectomy; Ax, axillary lymph node dissection; SLN, sentinel lymph-node; ER, estrogen receptor; PgR, progesterone receptor; LN, lymph-node involvement; CT, chemotherapy; HT, hormone therapy; RT, irradiation therapy; HER2, human epidermal growth factor receptor type 2; (-), no surgical treatment; +, positive; -, negative.

Table II. Statistics of the mEHT treatments.

\begin{tabular}{ccccc}
\hline Case & Total mEHT, $\mathrm{mEHT} / \mathrm{w}, \mathrm{n}$ & mEHT period, weeks & mEHT dose, $\mathrm{kJ}$ \\
\hline 1 & 36 & 3 & 12 & 13,464 \\
2 & 90 & 3 & 30 & 33,660 \\
3 & 47 & 2 & 23.5 & 17,578 \\
4 & 87 & 2 & 43.5 & 32,538 \\
5 & 73 & 3 & 24.3 & 27,302 \\
6 & 46 & 2 & 23 & 17,204 \\
7 & 8 & 2 & 4 & 2,992 \\
8 & 40 & 2 & 20 & 14,960 \\
9 & 15 & 2 & 7.5 & 5,610 \\
10 & 44 & 2 & 22 & 16,456 \\
\hline
\end{tabular}

Total mEHT, total number of mEHT; mEHT/w, number of mEHT per week; mEHT period, periods of mEHT (weeks); mEHT dose, total doses of mEHT (kj); mEHT, modulated electro-hyperthermia.

dendritic cell vaccine therapy were then performed; however, tumor growth was observed. At her request, mEHT alone was performed for 24 weeks and 73 times without chemotherapy. The symptoms of cough and dyspnea gradually worsened, and mEHT was discontinued due to difficulty in visiting the hospital. One month later, the patient died of cancerous pleurisy. Case 7 was a 75-year-old man with skin metastasis, lung. Preoperative chemotherapy was performed for stage IV breast cancer. Although lung metastasis was reduced, skin metastasis did not change. Eight sessions of mEHT+radiation therapy were performed, and a reduction in skin metastasis was observed (PR). Following treatment, he was recommended to undergo surgery but refused. Two months after the follow-up, chest CT revealed an exacerbation of lung metastases. Although anticancer drug treatment was restarted, progressively worsening lung metastases and dyspnea were observed. The patient eventually died of cancerous pleurisy 6 months after the completion of mEHT.
mEHT monotherapy. A total of 4 patients were treated with mEHT alone, following their request. As a result, one patient showed PR, two showed SD, and one showed PD. Case 3 had undergone breast cancer surgery and postoperative chemotherapy 22 years ago, and a recurrence of lung metastases was observed 19 years later. Hormone therapy was continued; however, an exacerbation of lung metastases was observed. Nevertheless, this time, the patient refused to receive anticancer drug treatment and only mEHT was performed 47 times. During that time, chest CT revealed no exacerbation of lung metastases; therefore, the patient was judged to be SD. Case 5 had undergone breast cancer surgery 5 years ago; 2 years later, she was diagnosed with liver, bone and lymph node metastases and received chemotherapy, hormone therapy and activated dendritic cell therapy. This time, the patient refused to receive anticancer drug treatment; therefore, only mEHT was performed 73 times. During that time, the level of the tumor marker CEA was elevated and an abdominal CT 
Table III. Complementary therapies and local responses.

\begin{tabular}{ccccc}
\hline Case & Combination & Response & CEA1, ng/ml & CEA2, ng/ml \\
\hline 1 & TDM-1 & PR & 8.1 & 5.1 \\
2 & mTOR, PTX+BV & PD & 292.8 & 4.5 \\
3 & $(-)$ & SD & 3.6 & 269.8 \\
4 & mTOR, Erib., PTX+BV & PD & 115.1 & 12.9 \\
5 & $(-)$ & PD & 145.5 & 45.1 \\
6 & mTOR & PD & 3.7 & 3.8 \\
7 & irradiation & PR & 3.2 & 3.5 \\
8 & $(-)$ & SD & 3.9 & 4.0 \\
9 & AI, Fulvestrant & SD & 10.6 & 2.1 \\
\hline
\end{tabular}

mTOR, mammalian target of rapamycin; TDM-1, Trastuzumab emtansine; Erib, Eribulin; PTX, Pacritaxel; BV, Bevasitsuzumab; AI, Aromatase inhibitor; PR, partial remission; SD, stable disease; PD, progressive disease; CEA, Carcinoembryonic antigen; (-), no combination therapy.

revealed aggravation of liver metastases; therefore, the patient was judged as PD. Case 8 had multiple lung metastases on preoperative chest CT; however, the patient refused any treatment other than surgery; therefore, only mEHT was performed 40 times after mastectomy. During the treatment period, no obvious subjective symptoms were observed and chest CT revealed no exacerbation of lung metastases. Therefore, the patient was considered to be SD. Details regarding the status of case 10 are provided later.

Statistical evaluation of mEHT. Univariate analysis of the number of various treatments performed before mEHT and their therapeutic effects are shown in Table IV. PD patients received more types of treatments before mEHT than PR+SD patients. CEA levels before and after mEHT were significantly higher in $\mathrm{PD}$ patients than in $\mathrm{PR}+\mathrm{SD}$ patients $(\mathrm{P}=0.017,0.009)$, and $\mathrm{mEHT}$ was performed in patients with more advanced cancer. Statistical analysis of the various parameters of mEHT and their therapeutic effects are shown in Table IV. The average number of treatments for $\mathrm{PR}+\mathrm{SD}$ patients (6 cases) was 31.6 times, and the treatment period 14.8 weeks, which was significantly less than that for PD cases (number of treatments, 74.0; treatment period, 30.2 weeks; $\mathrm{P}=0.002$ ). There were many advanced cancer patients with PD, and mEHT was often performed in combination with chemotherapy $(75 \%)$; however, no clear mEHT-related side effects were observed, and treatment for long periods was possible.

Clinical estimation of the PR cases. Showing the details, 2 PR cases are described. The PR cases 1, 7 and 10 had progression-free survival rates of 2, 7 and 9 months, respectively.

Case 1. Seven years ago, a 58-year-old woman visited our hospital due to left breast cancer recurrence. The TNM classification was T1N1M0 stage IIA at that time. Breast-conserving operation and additional dissection of left axillary lymph nodes were performed. However, the patient (then aged 65 years old) developed lung, skin and lymph node metastases. She was positive for the expression of HER2, ER and PgR. Postoperative radiation therapy (55 Gy) was performed on
Table IV. Results of statistical analysis.

\begin{tabular}{lccc}
\hline Factor & PR+SD & PD & P-value \\
\hline Age, years & 63.6 & 63.3 & 0.953 \\
Stage & & & 0.737 \\
1 & 0 & 1 & \\
2 & 3 & 1 & \\
3 & 1 & 1 & \\
4 & 2 & 1 & \\
Total mEHT, n & 31.6 & 74.0 & 0.006 \\
mEHT/w, n & 2.0 & 2.5 & 0.312 \\
mEHT period, weeks & 14.8 & 30.2 & 0.002 \\
mEHT dose, kJ & 11,843 & 27,676 & 0.002 \\
Pre-treat, total & 3.5 & 6.0 & 0.199 \\
Pre-CT, n & 1.8 & 2.5 & 0.689 \\
Pre-CEA, ng/ml & 5.51 & 141.5 & 0.017 \\
Post-CEA, ng/ml & 3.85 & 285.6 & 0.009 \\
\hline
\end{tabular}

Total mEHT, total number of mEHT; mEHT/w, number of mEHT per week; mEHT period, periods of mEHT (weeks); mEHT dose, total doses of mEHT $(\mathrm{kj})$; pre-treat, number of treatments before mEHT; pre-CT, number of chemotherapies before mEHT; pre-CEA, CEA before of mEHT; post-CEA, CEA after mEHT; PR, partial remission; SD, stable disease; PD, progressive disease; mEHT, modulated electro-hyperthermia.

the left residual breast tumor area, and 50 Gy radiotherapy on the left clavicular region. Hormone therapy (aromatase inhibitor) was continued after the completion of radiation therapy. A fluorodeoxyglucose-positron emission tomography scan revealed left chest wall skin invasion (or metastasis). Left cervical, subclavian and right axillary lymph node metastases were also observed. Although intravenous chemotherapy of trastuzumab was administered, metastatic skin lesions did not respond to these treatments. Combination chemotherapy with trastuzumab, pertuzumab and docetaxel was administered; however, intolerable diarrhea occurred. Since an exacerbation 
A

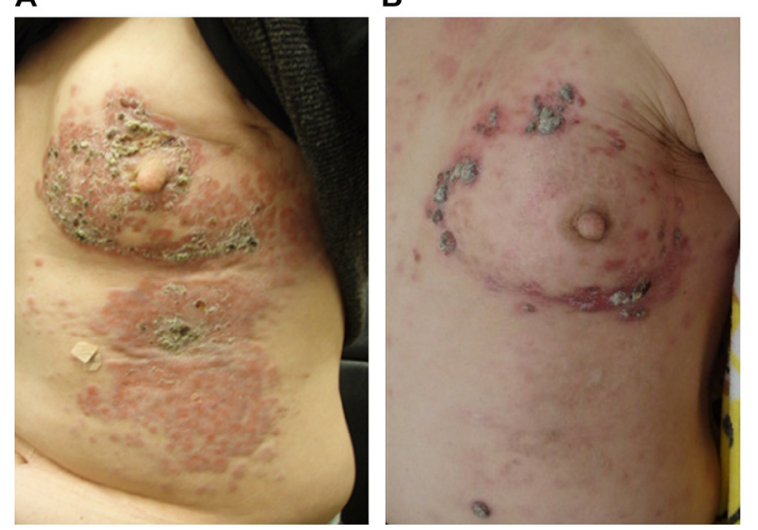

Figure 1. A representative case. (A) Exacerbation of skin metastasis from the breast cancer is presented before mEHT treatment. (B) Skin lesions responded very well to the treatment, whereas the tumor invasively penetrated into the large pectoral muscle. mEHT, modulated electro-hyperthermia.

of skin metastasis was observed after this treatment, the drug was changed to TDM-1. However, there was no improvement in the skin lesions (Fig. 1A). Finally, mEHT was used for adjuvant therapy using TDM-1. As a result of the combination of anticancer drug treatment (TDM-1) once every 3 weeks and mEHT thrice a week, a marked improvement in skin invasion and metastases was observed (Fig. 1B). During mEHT, right axillary lymph node metastasis was also reduced without direct intervention. However, the tumor re-increased after 2 months of post-treatment evaluation. The tumor metastasized to the brachial plexus. The patient was alive with disease 1.2 years after the final mEHT treatment.

Case 10. A 71-year-old woman had observed the presence of a mass in her right breast for $>15$ years but decided to ignore it. Two years ago, she was referred to our university hospital for the assessment of apparent discharge and bleeding from the protruding right breast mass. The definite diagnosis was breast cancer. The patient was recommended to undergo chemotherapy, hormone therapy and radiation therapy, but she rejected these treatment plans, out of fear of developing adverse effects. Therefore, she was followed up without any treatment. However, after the tumor increased in size with exudation and a foul-smelling odor, she accepted mEHT monotherapy. At the start of mEHT, an initial blood test showed a CEA level of $10.4 \mathrm{ng} / \mathrm{ml}$ and cancer antigen 15-3 (CA 15-3) of $132 \mathrm{U} / \mathrm{ml}$. $\mathrm{CT}$ and magnetic resonance imaging revealed the presence of a massive tumor measuring $15 \mathrm{~cm}$ in diameter in the right breast (Fig. 2A). Swelling of the axillary lymph nodes was also observed; however, distant metastasis to other organs was not detected. mEHT therapy was continued twice a week for 6 months, resulting in tumor shrinkage, as observed by CT; therefore, the patient was judged to have achieved PR (Fig. 2B). The preoperative diagnosis was $\mathrm{T} 4 \mathrm{cN} 3 \mathrm{bM} 0$ stage IIIB, which was an indication for right mastectomy (combined resection of the chest skin and partial large pectoral muscle), right axillary dissection, and second-stage skin transplantation. Intraoperative findings revealed that infiltration into the large pectoral muscle was mild and that it was possible to avoid total resection of the chest muscle. The skin with changed color was excised, and the tumor resection margin was histologically
A

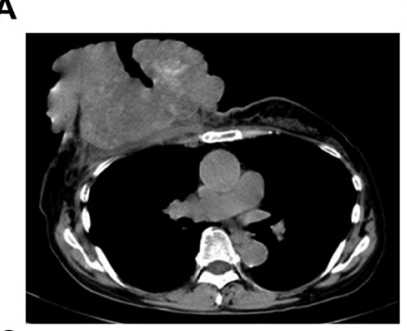

B
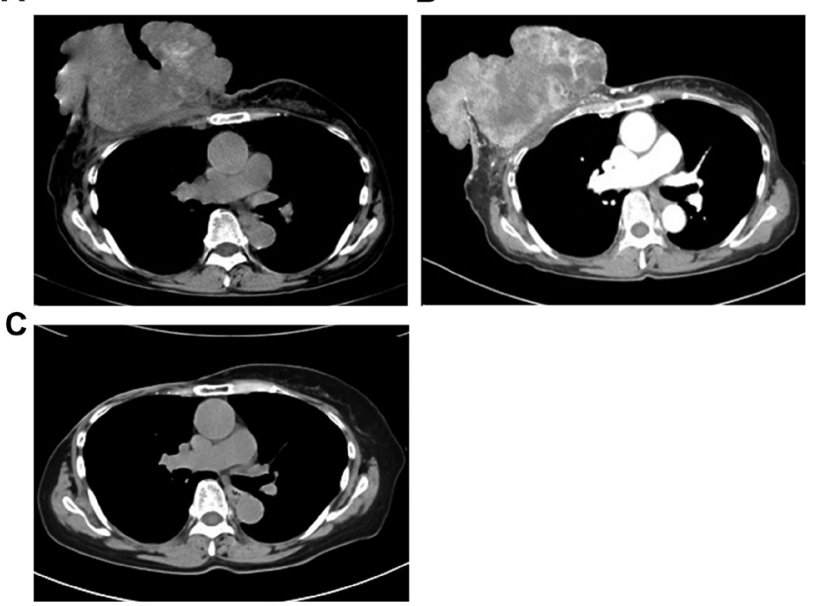

Figure 2. A second representative case. (A) CT showing the presence of a large tumor $15 \mathrm{~cm}$ in diameter. (B) Following mEHT treatment (twice a week), the tumor reduced in size after 6 months. (C) The tumor was removed at the curative margin due to the effectiveness of mEHT. Ten months after the surgery, the patient exhibit no evidence of disease. mEHT, modulated electro-hyperthermia.

negative. The axillary lymph nodes were dissected to level II, and it was evaluated that only level I lymph node was positive for metastasis. The postoperative course was unremarkable, and she was discharged on postoperative day 14 . The pathological diagnosis of the resected specimen was pT3N1 (level I, 2/24; level II, 0/14; level III, 0/2) M0 stage IIIA. The tumor was removed at the curative margin, due to the effectiveness of mEHT. After 3 weeks, the artificial dermis was affixed to the mastectomy part and grafting was performed from the thigh part of the patient. Postoperatively, the tumor did not reccur. The CEA level normalized to $2.1 \mathrm{ng} / \mathrm{ml} 1 \mathrm{month}$ after the surgery. CA15-3 also normalized to $18.6 \mathrm{U} / \mathrm{ml} 3$ months after the surgery. Nine months after the surgery, she showed no evidence of the disease (Fig. 2C).

\section{Discussion}

As the general lifestyle of people changes, the type and structure of malignant diseases also changes. The clinical course of cancer and its treatments have diversified. Furthermore, the growing of available open-access information has allowed patients to select their preferred therapies. The widely published adverse effects deter some individuals from receiving conventional therapies and favor conservative treatments with the hope of maintaining a normal life despite the occurrence of cancer. Hyperthermia is considered a less aggressive antitumor treatment strategy and sometimes could be applied even in patients who are unresponsive to conventional treatments (surgery, radiation or chemotherapy), as well as to new cancer immunotherapies, such as checkpoint inhibitors, cancer-specific cytotoxic $\mathrm{T}$ lymphocytes or chimeric antigen receptor-T-cell therapy.

In general, cancer cells proliferate autonomously and randomly. The cytoskeleton and genomic structure of malignant cells have an inherent instability; therefore, they are more sensitive to heat than normal cells (16). Utilizing this feature, the concept of hyperthermia has been established and various 
therapeutic approaches have been developed (17), including heating the lesion isothermally. Most hyperthermia methods use bio-electromagnetic energy-absorption heating of the cancer tissue of up to $43^{\circ} \mathrm{C}$ or higher temperatures to kill them, mainly by inducing local necrosis, such as the hyperthermia dose (CEM43 ${ }^{\circ} \mathrm{CTx}$ ) calibrated in vitro. Moreover, many experimental studies have shown that the obviously heterogenic solid tumors and their blood flow derail the developed temperature distribution, despite the use of iso-dose focusing. The usual vasodilatation that occurs in the vivid part of the tumor and its healthy neighborhood increases blood flow, possibly facilitating the delivery of chemotherapeutic drugs and increasing the reaction rate, as well improving the efficacy of ionization radiation therapies by delivering oxygen (18). Despite the advantages of high blood flow, it has several disadvantages, including delivering nutrients that support tumor growth and helping the dissemination of the malignant cells by the blood stream, thereby increasing the incidence of distant metastases (19). On the other hand, the heavily developed tumors have neo-angiogenetic vessels that form vasocontraction, increasing the severity of hypoxia and assisting rapid temperature growth in that part of the tissue $(20,21)$. This is the reason why local control is significantly good following the use of this method; however, overall survival is decreased due to metastases (22-26).

Due to the complex physiological feedback and the attempt to re-establish thermal homeostasis by increasing blood flow, as well as by other methods, the effects of conventional hyperthermia are not stable and mostly insufficient for a lifetime increase in blood flow. At the end of the 20th day of treatment, the clinical results for local control following radiation therapy alone vs. treatment with radiation+hyperthermia for advanced, recurrent breast cancer were reported to be 41 vs. 59\%, respectively (26). Therefore, many patients and medical doctors who continue to treat various types of cancer, including advanced pancreatic cancer and other advanced cases, without further conventional treatment options are looking for a more effective therapeutic method, including the safe and secure hyperthermia treatment. Based on these backgrounds, mEHT is conducted in accordance with basic and clinical research data, which is based on the cellular selection of tumor cells, inducing programmed cell death (apoptosis), in various cancer cells by causing a temperature gradient and prompting extrinsic pathways to produce damage-associated molecular patterns $(27)$ and immunogenic cell death $(28,29)$, thereby producing tumor-specific immune reactions (30) and an abscopal effect (31). The inhibition of protective autophagy via sublethal hyperthermia in hepatocellular carcinoma has been shown to enhance hyperthermia-induced apoptosis via the ATP/AMPK/mTOR signaling pathway (32). Furthermore, it has been reported that the inhibition of protective autophagy could be a therapeutic strategy for RAS-induced pancreatic cancer (33). Unfortunately, the combination therapy with mTOR inhibitor and mEHT used in the present study resulted in PD in all cases; however, it is possible to continue long-term treatment for advanced breast cancer cases with multiple organ metastases. More studies with more cases are needed to explore the combined treatment of mTOR inhibitor and mEHT. The following clinical advantages have been reported from this therapeutic principle: i) Very high heating efficiency for cancer with a low power $(150 \mathrm{~W})(34)$; ii) modulated electromagnetic waves do not result in burns on the skin (35), and (3) these waves adequately reach tumors deep within the body, such as those in the pancreas (36), lung (37), liver (32) and cervix (38).

Due to the lack of awareness and delay in discovery, elderly patients with breast cancer are sometimes at a very progressive stage, with skin invasion or other metastases upon first diagnosis. Therefore, it is essential to consider the risks and benefits of surgery and anticancer drug treatment for these patients. When conventional therapies with standard protocols fail, only palliative care is selected after informed consent. However, mEHT is recommended as a valid option with few adverse effects for patients with advanced cancer.

In conclusion, it was reported in the present study that the use of mEHT is feasible for advanced or recurrent metastatic breast cancer where pretreatment is ineffective. The results suggested that mEHT has no side effects and could be combined with various treatments for a long time.

\section{Acknowledgements}

The authors would like to thank Professor Takashi Kondo (Division of Radiation Oncology, Department of Radiology, Faculty of Medicine, University of Toyama, Toyama, Japan) for providing medical advice for this research. The authors would also like to thank Mrs. Nagisa Isobe (Division of Oncothermia, Department of Human Science, Faculty of Medicine, University of Toyama, Toyama, Japan) for technical support in operating the mEHT system.

\section{Funding}

The current study was funded by Teteyamma Machine Co. (Toyama, Japan).

\section{Availability of data and materials}

The datasets used and/or analyzed during the current study are available from the corresponding author on reasonable request.

\section{Authors' contributions}

MK conceived and designed the study. SS, MA and MM acquired the data. TN and TF analyzed and interpreted the data. TN drafted the manuscript. MK, SS and TF performed critical revision of the manuscript. All authors read and approved the final manuscript.

\section{Ethics approval and consent to participate}

All experiments were approved by the Ethics Committee of the University of Toyama (Toyama, Japan; approval no. 26-13), and written informed consent was obtained from all participants.

\section{Patient consent for publication}

All patients provided written consent for the publication of their data and images. 


\section{Competing interests}

The authors declare that they have no competing interests.

\section{References}

1. Tremont A, Lu J and Cole JT: Endocrine therapy for early breast cancer: Updated review. Ochsner J 17: 405-411, 2017.

2. Balaji K, Subramanian B, Yadav P, Radha CA and Ramasubramanian V: Radiation therapy for breast cancer: Literature review. Med Dosim 41: 253-257, 2016.

3. Cody HS III: Current surgical management of breast cancer. Curr Opin Obstet Gynecol 14: 45-52, 2002.

4. Ito Y: Chemotherapy and hormone therapy for breast cancer: Current status and perspective. JMAJ 45: 424-433, 2002.

5. Munagala R, Aqil F and Gupta RC: Promising molecular targeted therapies in breast cancer. Indian J Pharmacol 43: 236-245, 2011

6. Vincze G, Szasz O and Szasz A: Generalization of the thermal dose of hyperthermia in oncology. Open J Biophys 5: 97-114, 2015

7. Roussakow S: The History of Hyperthermia Rise and Decline. Hindawi Publishing Corporation Conference Papers in Medicine. Vol 2013, ID428027, 2013. http://www.hindawi.com/ archive/2013/428027/.

8. Szasz A, Iluri N and Szasz O: Local hyperthermia in oncology-to choose or not to choose? In: Hyperthermia. Huilgol N (ed) InTech, Croatia, pp1-82, 2013.

9. Szasz O, Szasz AM, Minnaar C and Szasz A: Heating-trends in modern oncological hyperthermia. Open J Biophys 7: 116-144, 2017.

10. Szasz A: Electromagnetic effects in nanoscale range. In: Cellular Response to Physical Stress and Therapeutic Applications, cheptor 4. Shimizu T and Kondo T (eds). Nova Science Publishers, New York, NY, pp55-81, 2013.

11. Papp E, Vancsik T, Kiss E and Szasz O: Energy absorption by the membrane rafts in the modulated electro-hyperthermia (mEHT). Open J Biophys 7: 216-229, 2017.

12. Szasz A, Szasz N and Szasz O: Oncothermia-principles and practices. Springer Science, Heidelberg, 2010.

13. Lee SY and Lee NR: Positive response of a primary leiomyosarcoma of the breast following salvage hyperthermia and pazopanib. Korean J Intern Med 33: 442-445, 2018.

14. Schirrmacher V, Stücker W, Lulei M, Bihari AS and Sprenger T: Long-term survival of a breast cancer patient with extensive liver metastases upon immune and virotherapy: A case report. Immunotherapy 7: 855-860, 2015

15. Anjum F, Razvi N and Massod MA: Breast cancer therapy: A mini review. MOJ Drug Des Develop Ther 1: 35-38, 2017.

16. Dewey WC, Hopwood LE, Sapareto SA and Gerweck LE: Cellular responses to combinations of hyperthermia and radiation. Radiology 123: 463-474, 1977

17. Smythe WR and Mansfield PF: Hyperthermia: Has its time come? Ann Surg Oncol 10: 210-212, 2003.

18. Oleson JR: Eugene robertson special lecture, hyperthermia from the clinic to the laboratory: A hypothesis. Int J Hyperthermia 11: 315-322, 1995

19. Mitsumori M, Zeng ZF, Oliynychenko P, Park JH, Choi IB, Tatsuzaki H, Tanaka Y and Hiraoka M: Regional hyperthermia combined with radiotherapy for locally advanced non-small cell lung cancers: A multi-institutional prospective randomized trial of the international atomic energy agency. Int J Clin Oncol 12: 192-198, 2007.

20. Reinhold HS and Endrich B: Tumor microcirculation as a target for hyperthermia. Int J Hyperthermia 2: 111-137, 1986.

21. Song CW: Effect of local hyperthermia on blood-flow and microenvironment: A review. Cancer Res 44 (Suppl 10): 4721s-4730s, 1984

22. Kay CS, Choi IB, Jang JY, Choi BO, Kim A and Shinn KS Thermoradiotherapy in the treatment of locally advanced nonsmall cell lung cancer. J Korean Soc Ther Radiol Oncol 14 115-122, 1996.

23. Zolciak-Siwinska A, Piotrkowicz N, Jonska-Gmyrek J, Nicke-Psikuta M, Michalsky W, Kawczyńska M, Bijok M and Bujiko K: HDR brachytherapy combined with interstitial hyperthermia in locally advanced cervical cancer patients initially treated with concomitant radiochemotherapy-a phase III study. Radiother Oncol 109: 194-199, 2013.
24. Jones EL, Oleson JR, Prosnitz LR, Samulski TV, Vujaskovic Z, Yu D, Sanders LL and Dewhirst MW: Randomized trial of hyperthermia and radiation for superficial tumors. J Clin Oncol 23 3079-3085, 2005

25. Sherar M, Liu FF, Pintilie M, Levin W, Hunt J, Hill R, Hand J, Vernon C, van Rhoon G, van der Zee J, et al: Relationship between thermal dose and outcome in thermoradiotherapy treatments for superficial recurrences of breast cancer: Data from a phase III trial. Int J Radiat Oncol Biol Phys 39: 371-380, 1997.

26. Vernon CC, Hand JW, Field SB, Machin D, Whaley JB, van der Zee J, van Putten WL, van Rhoon GC, van Dijk JD, González González D, et al: Radiotherapy with or without hyperthermia in the treatment of superficial localized breast cancer: Results from five randomized controlled trials. International collaborative hyperhtermia group. Int J Radiat Oncol Biol Phys 35: 731-744, 1996.

27. Forika G, Balogh A, Vancsik T, Zalatnai A, Petovari G, Benyo Z and Krenacs T: Modulated electro-hyperthermia resolves radioresistance of Panc1 pancreas adenocarcinoma and promotes DNA damage and apoptosis in vitro. Int J Mol Sci 21: 5100, 2020

28. Hurwitz MD: Hyperthermia and immunotherapy: Clinical opportunities. Int J Hyperthermia 36 (Suppl 1): S4-S9, 2019.

29. Huang L, Li Y, Du Y, Zhang Y, Wang X, Ding Y, Yang Y, Meng F, Tu J, Luo L and Sun C: Mild photothermal therapy potentiates anti-PD-L1 treatment for immunologically cold tumors via an all-in-one and all-in-control strategy. Nat Commun 10: 4871, 2019.

30. You SH and Kim S: Feasibility of modulated electro-hyperthermia in preoperative treatment for locally advanced rectal cancer: Early phase 2 clinical results. Neoplasma 67: 677-683, 2020.

31. Minnaar CA, Kotzen JA, Ayeni OA, VAngu MD and Bawyens A: Potentiation of the abscopal effect by modulated electro-hyperthermia in locally advanced cervical cancer patients. Front Oncol 10: 376, 2020.

32. Jiang J, Chen S, Li K, Zhang C, Tan Y, Deng Q, Chai Y, Wang X, Chen G, Feng K, et al: Targeting autophagy enhances heat stress-induced apoptosis via the ATP-AMPK-mTOR axis for hepatocellular carcinoma. Int J Hyperthermia 36: 499-510, 2019.

33. Kinsey CG, Camolotto SA, Boespflug AM, Gillen KP, Foth M, Truong A, Schuman SS, Shea JE, Seipp MT, Yap JT, et al: Protective autophagy elicited by $\mathrm{RAF} \rightarrow \mathrm{MEK} \rightarrow$ ERK inhibition suggests a treatment strategy for RAS-driven cancers. Nat Med 25: 620-627, 2019.

34. Van Gool SW, Makalowski J, Feyen O, Prix L, Schirrmacher V and Stuecker W: The induction of immunogenic cell death (ICD) during maintenance chemotherapy and subsequent multimodal immunotherapy for glioblastoma (GBM). Austin Oncol Case Rep 3: 1-8, 2018.

35. Szasz AM, Minnaar CA, Szentmártoni G, Szigeti GP and Dank M: Review of the clinical evidences of modulated electro-hyperthermia (mEHT) method: An update for the practicing oncologist. Front Oncol 9: 1012, 2019.

36. Fiorentini G, Sarti D, Casadei V, Milandri C, Dentico P, Mambrini A, Nani R, Fiorentini C and Guadagni S: Modulated electro-hyperthermia as palliative treatment for pancreatic cancer: A retrospective observational study on 106 patients. Integr Cancer Ther 18: 1534735419878505, 2019.

37. Ou J, Zhu X, Chen P, Du Y, Lu Y, Peng X, Bao S, Wang J, Zhang X, Zhang T and Pang CLK: A randomized phase II trial of best supportive care with or without hyperthermia and vitamin $\mathrm{C}$ for heavily pretreated, advanced, refractory non-small-cell lung cancer. J Adv Res 24: 175-182, 2020.

38. Minnaar CA, Kotzen JA, Ayeni OA, Naidoo T, Tunmer M, Sharma V, Vangu MD and Baeyens A: The effect of modulated electro-hyperthermia on local disease control in HIV-positive and -negative cervical cancer women in South Africa: Early results from a phase III randomised controlled trial. PLoS One 14: e0217894, 2019.

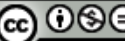

This work is licensed under a Creative Commons Attribution-NonCommercial-NoDerivatives 4.0 International (CC BY-NC-ND 4.0) License. 